\section{Festrede anlässlich der Diplomfeier der medizi- nischen Fakultät Bern, 21. März 2000}

Dr. René Salzberg

Präsident des Leitenden Ausschusses für die eidg. Medizinalprüfungen Mitglied des Zentralvorstandes der FMH

Sehr geehrter Herr Dekan,

sehr geehrte Damen und Herren Professoren,

sehr geehrte Kolleginnen und Kollegen,

sehr geehrte Gäste

Es ist für mich eine grosse Ehre, auf Einladung Ihres Dekans heute hier zu Ihnen sprechen zu dürfen.

Ich tue dies mit einem etwas schlechten Gewissen. Erstens bin ich seit vielen Jahren gegen das Übermass an Frontalvorlesungen angetreten, mit dem die meisten Medizinstudierenden vor allem in den ersten Studienjahren geplagt wurden, und jetzt bin ich im Begriffe, eben dasselbe zu tun. Und zweitens werde ich Ihnen einige Minuten Ihrer Zeit wegnehmen. Und Zeit ist etwas ausserordentlich Wertvolles, das einzige, was Ihnen nie mehr jemand zurückgeben kann. Ich werde mich also bemühen, kurz zu sprechen, um nicht zuviel Ihrer (und auch meiner!) Zeit zu beanspruchen, und ich werde versuchen, nur Dinge $\mathrm{zu}$ sagen, welche mir wesentlich erscheinen.

Ich möchte die folgenden Minuten, welche ich Ihnen wegnehme, nutzen, um mich kurz vorzustellen und um Ihnen einige Überlegungen zu vermitteln $\mathrm{zu}$ einer Thematik, welche ich mir selbst vorgegeben habe.

Ich habe über 30 Jahre lang als Kinderarzt eine pädiatrische Praxis geführt, bin seit 1981 Mitglied des Zentralvorstandes der Verbindung der Schweizer Ärztinnen und Ärzte FMH und präsidiere dort die Kommission für Weiter- und Fortbildung. Seit 1989 bin ich Präsident des Leitenden Ausschusses für die eidg. Medizinalprüfungen, einer Behördenkommission, welche vom Bundesrat gewählt ist und welche die Aufgabe hat, die Inhalte des Studiums und das Prüfungswesen der vier traditionellen Medizinalberufe zu überwachen, nämlich der Humanmedizin, der Zahnmedizin, der Veterinärmedizin und der Pharmazie.

Warum erzähle ich Ihnen dies? Diese kurze persönliche berufliche Anamnese soll die äusseren Umstände schildern, den Rahmen, welcher mir ermöglicht hat, mich in den vergangenen 20 Jahren fast hauptberuflich intensiv mit Fragen der Aus-, Weiterund Fortbildung der Ärzteschaft zu befassen. Diese Beschäftigung wird in der Schweiz nicht dadurch erleichtert, dass entsprechend den Schweizer Gepflogenheiten die Kompetenzen für diverse Bereiche an völlig verschiedene Organisationen oder Gremien verteilt werden: für die Ausbildung an die Universitäten und ihre Fakultäten, für das Prüfungswesen an die Eidgenossenschaft, für die Weiterbildung und die Fortbildung an den Privatverein FMH. Die Voraussage, ob sich bei dieser Arbeit persönliche Erfolgserlebnisse einstellen werden, kann nicht mit Sicherheit gemacht werden. Viel eher kann sich der nicht direkt involvierte Beobachter fragen, wie hoch das Ausmass des persönlichen Masochismus ist und wie gut man sich in die Rolle eines Sisyphus einleben kann, will man sich mit der beschriebenen Thematik befassen!

Denken Sie immer daran: Die individuelle $\mathrm{Zu}-$ kunft ist erträumbar, aber nicht voraussehbar!

Beginnen wir mit der Ausbildung, dem Studium, und ich möchte mich im folgenden hauptsächlich auf dasjenige der Humanmedizin beschränken, weil ich dieses am besten kenne, nicht nur aus eigener, subjektiver und mit zunehmendem Alter auch "verklärter» Perspektive, sondern, weil auch meine zwei Söhne Jahre später durch die Mühlen des Medizinstudiums und der Prüfungen gegangen sind.

In der wunderbaren Ausstellung über die Geschichte und Kunst des antiken Syriens im Basler Antikenmuseum las ich in der Abteilung "Medizin" folgendes:

"Und in meiner Person findet Ihr den besten Barbier Bagdads, einen erfahrenen Arzt, einen erleuchteten Chemiker, einen Astrologen, der sich niemals irrt, einen vollendeten Grammatiker, einen glänzenden Redner, einen scharfen Logiker, einen Mathematiker mit besten Kenntnissen der Geometrie, der Arithmetik, der Astronomie und aller Details der Algebra, sowie einen Historiker, der die Geschichte aller Königreiche des Universums kennt, und all dies in einer Person vereinigt! Abgesehen davon bin ich ein Kenner der philosophischen Schriften, der Gesetze und sämtlicher Bräuche, ich bin Poet und Architekt!»

Diese Selbstbeschreibung eines Arztes stammt nicht aus Europa und auch nicht vom Kontinent der unbegrenzten Möglichkeiten, auch nicht aus Afrika oder Australien. Dieses blumig beschriebene Arztbild ist zitiert aus der wunderschönen Sammlung von Märchen und Erzählungen aus dem Orient, nämlich aus "1001 Nacht». So unrealistisch und phantastisch diese Beschreibung klingt, so malt sie doch ein gewisses Idealbild eines Arztes, das offenbar auch beim Kalifen von Bagdad angestrebt werden sollte, und umso mehr sollten wir deshalb dasjenige Arztbild hinterfragen, welches die medizinischen Fakultäten bei uns formen wollen.

Was waren und sind eigentlich die Gründe, derentwegen das Studium reformiert, das heisst verbessert werden soll? 
Ein Zitat, welches für mich die Begründungen für Reformen treffend wiedergibt, heisst: "There are only two lasting bequests we can hope to give our children. One of these is roots, the other wings." (Hodding Carter)

Waren oder sind die "roots", die Wurzeln, die richtigen? Ist es tatsächlich diejenige Physik oder Chemie, welche während Jahrzehnten von Lehrern der naturwissenschaftlichen Fakultäten gelehrt wurde, ist es eine bis vor kurzem traditionelle, um nicht $\mathrm{zu}$ sagen antiquierte und verstaubte Biologie, welche sich noch in vielen Stunden von Frontalunterricht mit Botanik, mit der vergleichenden Morphologie der Wirbeltiere, mit der Onto- und Phylogenese befasste? Ist es die morphologische Anatomie, wo man Knochenvorsprünge und -löcher, Einzelorgane für sich, und dann in weiteren Vorlesungen und Kursen, unabhängig davon und zeitlich nicht darauf abgestimmt, Funktionen und Biochemie lehrte? Ist es die Lehre von den normalen und gestörten Funktionen des menschlichen Körpers, welche losgelöst von praktischen Problemen und von einer zum mindesten virtuellen Realität verkündet wurde?

Ich weiss sehr gut, dass ich hier in Bern mindestens teilweise am falschen Orte predige. Die medizinische Fakultät Bern hat seit vielen Jahrzehnten versucht, mehr oder minder erfolgreich, Reformen einzuführen. Ja, ich darf behaupten, dass die wesentlichen Anstösse für die sich jetzt im Gang befindliche gesamtschweizerische Reform aus Bern gekommen sind: Prof. Hannes Pauli war es, welcher mich im Jahre 1989 dazu anregte, eine Studienreise dorthin $\mathrm{zu}$ organisieren, wo man nicht nur von Reformen sprach, sondern sie auch angefangen oder bereits beendet hatte, nämlich von den USA und Kanada.

Und so flogen wir dann im Jahre 1989, einige verbrannte Optimisten und Träumer aus Bern, Genf und Basel, nach Toronto, nach Boston, nach Montreal und nach Albuquerque und sahen uns unvoreingenommen an, was dort an den Universitäten geschah. Es war vor allem in den ersten Jahren des Studiums eine andere Welt: wir sahen Jahreskurse von begeisterten und engagierten Studenten, welche keine Vorlesung oder Kursstunde versäumen wollten, wir erlebten Gruppenunterricht, wo die Lehrer zu Schülern wurden, weil der Enthusiasmus der Studierenden manchmal fast überbordete und man erzählte uns, dass viele der späteren Nobelpreisträger an der renommierten Harvard-Universität von kleineren, weniger bekannten Medizinschulen gekommen waren, wo sie das "neue Studium", das sogenannt problemorientierte Lernen mit- und durchgemacht hatten.

Was waren die Erkenntnisse und Konsequenzen, welche sich für uns aus dieser Studienreise ergaben? Wir hatten gesehen, dass es "roots», Wurzeln, braucht. Es gibt keine Medizin ohne solide naturwissenschaftliche Kenntnisse. Aber eben: zur Naturwissenschaft gehören eben nicht nur Physik, Chemie und Biologie, sondern auch geisteswissenschaftliche, kulturelle, soziale und ethische Aspekte. Nur eine gute und praktisch anwendbare Durchmischung dieser Themen wird schliesslich die Qualität der Ärztin oder des Arztes verbessern.

Wir brauchen dringend neue Inhalte: die Kommunikationsfähigkeiten und die Beschäftigung mit den Sozialwissenschaften müssen in die Curricula einfliessen, diese wiederum müssen offen und flexibel gestaltet werden, die Forschung muss auch in die Lehre einfliessen und die neuen Technologien müssen im Umfeld der Lehre und bei der Informationsbeschaffung berücksichtigt werden.

In der akademischen Lehre sollte das sehr einfache Prinzip einer effizienten Entwicklungshilfe gelten: "Give them a fish and they have to eat for one day, teach them how to catch fish, and they have to eat all their life."

Und vor allem: die richtig gewählten Grundlagen, die an einer medizinischen Fakultät zu erreichenden Lehrziele, die "roots", werden es einer kommenden Generation von Ärztinnen und Ärzten ermöglichen, ihre "wings", ihre Flügel, auszubreiten und selbständig zu fliegen. Lebenslänglich ein Lernender zu bleiben! Die Kenntnisse, das Wissen, die Zusammenhänge bei neu sich stellenden Problemen anzuwenden zur Lösung theoretischer Probleme oder zum Wohle von Patientinnen und Patienten.

"A thought which does not result in an action is nothing much, and an action which does not result from a thought is nothing at all». (Georges Bernanos) Dieser Ausspruch zeigt uns klar, dass es eben "roots» braucht, um die "wings», die Flügel, zu benutzen.

Die Strukturen, die dadurch geleiteten Prozesse sind an manchen medizinischen Fakultäten veraltet, verkrustet. Sie müssen aufgebrochen werden, es braucht neuen Wind, es braucht eine Aufwertung des "teaching", der Lehre. Es braucht Lehrer, welche im Sinne einer Gesamtreform des Medizinstudiums und eines erneuerten Arztbilds in einer modernen Gesellschaft lehren und gewillt sind, sich selbst konstant in diesem Prozess in Frage zu stellen und sich den Herausforderungen zu stellen!

Fachhochschule oder Universität? Ich glaube, dass mit den eben gemachten Ausführungen diese Frage überflüssig wird. Die Grundlage muss für alle Möglichkeiten medizinischer und ärztlicher Tätigkeit gut genug sein, die Denkstrukturen und -prozesse sind es, welche den Arzt als Forscher, als praktizierenden Arzt, als Administrator oder als Industriearzt prägen!

Liebe junge Kolleginnen und Kollegen

Mit dem Arztdiplom, welches Sie mit einem langen Studium und mit dem Bestehen vieler Prüfungen erworben haben, sind Sie an einem ersten «Milestone» Ihrer beruflichen Karriere angekommen. Es gilt jetzt für Sie, erste Weichen zu stellen, denjenigen $\mathrm{Zug}$ zu wählen und zu besteigen, welcher Sie wahrscheinlich an das Ziel bringt, das Sie erreichen wollen. Die vor Ihnen stehende Phase der Weiterbildung wird das vertiefen, was Sie in Ihrem Grundstudium gelernt und sich angeeignet haben. Auch das beste Medizinstudium der Welt könnte Sie nicht ausreichend auf Ihre zukünftige Tätigkeit als Ärztinnen und Ärzte vorbereiten, dafür braucht es zusätzlich einige Jahre gut strukturierter und geplanter Weiterbildung an Insti- 
tuten und Kliniken. Voraussichtlich werden 80 bis 90\% von Ihnen eine patientenbezogene Tätigkeit als Ärzte ausüben. Dieser Prozentsatz ist interessanterweise auf der ganzen Welt bei den Abgängern von Medizinschulen derselbe. Wahrscheinlich liegt die Begründung darin, dass Sie ja eben gerade darum Medizin studiert haben, um später einmal den ärztlichen Beruf auszuüben.

Die Verbindung der Schweizer Ärztinnen und Ärzte FMH ist seit über 60 Jahren die Verwalterin und Garantin der Weiterbildung der Schweizer Ärzteschaft. Sie erarbeitet zusammen mit den medizinischen Fachgesellschaften und Fakultäten die Weiterbildungsprogramme, welche in der Regel fünf bis sieben Jahre dauern und die nach dem Bestehen einer Facharztprüfung zum Erwerb eines Facharzttitels führen. Ohne einen Facharzttitel, sei dies nun ein Spezialarzt oder ein Allgemeinarzt, ist heutzutage eine verantwortungsvolle selbständige ärztliche Tätigkeit nicht mehr denkbar. Deshalb fordert der Bundesrat im neuen revidierten Freizügigkeitsgesetz auch den Besitz eines Facharzttitels als Voraussetzung für das selbständige Führen einer Praxis. Die FMH bemüht sich um eine ständige Optimierung der Weiterbildungsperiode. Ich rufe Sie dazu auf, als Assistentinnen und Assistenten bei diesen Bestrebungen mitzuhelfen, indem Sie bei den alljährlich durchgeführten Evaluationswochen Ihre Weiterbildungsstellen und deren Leiterinnen und Leiter kritisch-objektiv beurteilen und der FMH Ihre Beurteilungen, welche in anonymisierter Form erfolgen, zukommen zu lassen.

Und last but not least: die kontinuierliche ärztliche Fortbildung, welche nach einer definitiven beruflichen Etablierung in einer eigenen Praxis oder in einer Spitalposition einsetzt, das lebenslange Lernen nach der Phase der Weiterbildung. Das ständige Bestreben, die eigene ärztliche Kompetenz zu erhalten oder möglichst auch zu verbessern, muss uns während unserer beruflichen Aktivität begleiten. Der immer wieder zitierte Erfahrungsgewinn allein genügt heute bei weitem nicht mehr als Fortbildungsinstrument. Auch die weissen, blinden Flecke in unserem Kompetenzspektrum müssen Nahrung erhalten, auch wenn dies für manche nicht immer sehr angenehm und bequem ist! Die Fortbildungsordnung der FMH ist für eine strukturierte und nachweisbare Fortbildung ein gutes und brauchbares Instrumentarium, und hat, zum mindesten bis anhin, staatliche Eingriffe in diesem Gebiet verhindert.

Meine Damen und Herren

Ich kann Ihnen nachfühlen, wie froh Sie alle sind, Ihr Studium erfolgreich hinter sich gebracht $\mathrm{zu}$ haben. Eine Diplomfeier in festlichem Rahmen, zusammen mit Ihren Angehörigen, Freunden und akademischen Lehrern ist etwas Wunderschönes und Unvergessliches. Ich wünsche Ihnen alles Gute und viel Glück in ihrem privaten und beruflichen Leben.

Der portugiesische Dichter Fernando Pessoa schreibt in seinen "Poesias» folgendes:

Ich machte aus mir, was ich nicht verstand; und was ich machen konnte aus mir, liess ich bleiben. Der Domino, den ich anzog, verfing nicht. Man erkannte mich gleich als den, der ich nicht war; ich wehrte mich nicht und verlor mich.

Als ich die Maske abnehmen wollte, blieb sie mir im Gesicht kleben.

Denken Sie immer daran: Bleiben Sie sich selbst treu, ziehen Sie keine Maske an, die Ihnen nicht steht und die Sie selbst unzufrieden und unglücklich machen wird. Machen Sie aus sich, was Sie für richtig halten und wozu Ihr Intellekt und Ihre Gefühle Ihnen raten. Sie haben das Privileg, in einem der schönsten Berufe, den es gibt, tätig zu sein, einem Beruf, der es Ihnen ermöglicht, Menschen zu beraten und zu helfen durch Ihr Wissen und Können, aber vor allem durch Ihre Persönlichkeit und Zuwendung. 


\section{Facharztprüfung zur Erlangung des Facharzttitels FMH für klinische Pharmakologie}

Aufgrund des Weiterbildungsprogrammes, welches am 1. Juli 1995 in Kraft gesetzt wurde, ist die Teilnahme an der Facharztprüfung für diejenigen Kandidatinnen und Kandidaten Voraussetzung für die Erlangung des Facharzttitels FMH für klinische Pharmakologie, welche ihre Weiterbildung in klinischer Pharmakologie am 30. Juni 1996 nicht abgeschlossen hatten. Es empfiehlt sich, die Facharztprüfung frühestens im letzten Jahr der reglementarischen Weiterbildung abzulegen (Art. 22 WB0).
Ort: wird nach Eingang der Anmeldungen bekanntgegeben.

Datum: Mittwoch, 20. Dezember 2000

Prüfungsgebühr: Die Fachgesellschaft erhebt eine Prüfungsgebühr von Fr. 400.-.

Anmeldung und Information:

Sekretariat SKP, Herr Dr. med. H. Kupferschmidt, Schweiz. Toxikologisches Informationszentrum, Freiestrasse 16, 8028 Zürich,

Tel. 0163410 20, Fax 012528833

Anmeldefrist: 31. August 2000

\section{Examen de spécialiste en vue de l'obtention du titre de spécialiste FMH en pharmacologie clinique}

Conformément au programme de formation postgraduée entré en vigueur le $1^{\text {er }}$ juillet 1995 , la participation à l'examen de spécialiste est obligatoire pour les candidats à l'obtention du titre de spécialiste FMH en pharmacologie clinique qui n'avaient pas terminé leur formation postgraduée en pharmacologie clinique le 30 juin 1996. Il leur est recommandé de passer l'examen de spécialiste au plus tôt durant la dernière année de leur formation postgraduée réglementaire (art. 22 RFP).
Lieu: sera communiqué après réception des inscriptions.

Date: mercredi, 20 décembre 2000

Taxe d'examen: la société de discipline médicale prélève une participation aux frais de Fr. 400.-.

Inscription et information:

Secrétariat SPC, Dr H. Kupferschmidt,

Centre Suisse d'Information toxicologique,

Freiestrasse 16, 8028 Zurich,

tél. 0163410 20, fax 012528833

Délai d'inscription: 31 août 2000 


\section{Corrigendum}

\section{Examen de spécialiste pour l'obtention du titre FMH en cardiologie}

Conformément au programme de formation postgraduée et à la décision du Comité central de la FMH du 3 février 1999, la réussite de l'examen de spécialiste est une condition requise pour les candidats au titre FMH en cardiologie qui termineront leur formation postgraduée après le 31 décembre 2000. Pour de plus amples renseignements concernant les dispositions transitoires et les dérogations, veuillez vous référer à la publication du Bulletin des médecins suisses no 5, du 2 février 2000.
Lieu: Centre de Division de Cardiologie, HUG, Genève

Date: Jeudi 7 décembre 2000, à $8 \mathrm{~h}$

Taxe d'examen: La SSC prélève une participation aux frais de Fr. 500.-.

Inscriptions (avec soumission du curriculum vitae): Prof. Alberto Righetti, Centre et Division de Cardiologie, Hôpital Cantonal, 1211 Genève 14, tél. 02237272 02, fax 0223727229

Délai d'inscription: 30 septembre 2000 